\title{
ISCRIZIONI ETRUSCHE DI DEDICA: ENFASI DEITTICA PRONOMINALE E MARCATEZZA ANAFORICA VERBALE
}

\author{
DOMENICO SILVESTRI (*)
}

RIASSUNTO. - Sulla base di un'ampia raccolta di testi riconoscibili come iscrizioni di dedica si cercherà di risolvere $\mathrm{o}$, almeno, dare una nuova impostazione al problema di alcune forme verbali presunte come "perfettive" (alice, turuce, mulvanice, etc.) che non presentano un rapporto morfologico simmetrico rispetto alle presunte forme "participiali" e/o "nominali corrispondenti (aliqu, turu, mulu, etc.). Si insisterà inoltre sui limiti delle pretese "traduzioni" di testi etruschi per quanto concerne la sfera pragmalinguistica dell'offerta, della dedica, del dono (e di altro ancora), mostrando che le rese lessicali si propongono di fatto come varianti pseudosinonimiche di un iperonimo generico del trasferimento di un oggetto da un'entità emittente ad una ricevente. Sarà ripreso sotto nuova luce il problema dell'opposizione morfologica tra forme "attive" in -ce e forme "passive" in -khe, svincolando fin dove è possibile l'etrusco da una percezione tipologica "paraflessiva" (contesto indoeuropeo dell'Italia antica) a favore di un'agnizione tipologica genericamente "agglutinante" (e presuntivamente asianica, con implicita riproposizione del problema delle "origini etrusche").

$* * *$

ABSTRACT. - Considering an extended collecting of texts recognized as dedication inscriptions we'll try to solve or, at least, to give a new formulation to the problem of some oral forms supposed to be "perfective" (alice, turuce, mulvanice, etc.) that don't introduce a symmetrical morphological relationship in comparison to the presumed "participial" e/o "nominal correspondent forms (aliqu, turu, mulu, etc.). We will also insist on the limits of the pretented "translations" of Etruscan texts for what the pragmalinguistics sphere of the offer, of the dedication, of the gift (etc...) is concerned, showing that the lexical translations are actually proposed as pseudosynonymic variations of a generic hyperonym of the transfer of an object from an emitting subject to a receiver. It will be taken under new light the problem of the morphological opposition among the "active" forms

(*) Università degli Studi di Napoli "L'Orientale”. 
in -ce and the "passive" forms in - khe, releasing as much as possible the Etruscan from a typological parainflected perception (indoeuropean context of Ancient Italy).

\section{OFFERTA, DEDICA, DONO (E ALTRO ANCORA)}

In questa sede proverò solo ad abbozzare alcune considerazioni preliminari (tra pragmatica e linguistica testuale) a proposito di una distinzione, teoricamente possibile ma solo processualmente legittimabile nello "spazio" etrusco della "dedica", tra "offerta" (di carattere religioso) e "dono" (di carattere laico). In via preliminare dichiarerò che proprio dal punto di vista qui assunto non bisogna sopravvalutare le premesse o presupposizioni lessicali, quale ad esempio la pretesa coppia paradigmatica muluvanice "dedicare (religioso)" - turuce "donare (laico)", ma non bisogna nemmeno sottovalutare le diverse epifanie storicolinguistiche e morfologiche: l'arcaico (solo VII e VI sec.) mulu(v)-ani-ce rispetto al non marcato mulu "dedica" presenta infatti una specifica marcatezza verbale, che è assente (o quasi del tutto assente) ${ }^{1}$ nel recenziore (a partire dalla fine del VI sec.) turu-ce rispetto al non marcato turu "dono".

\section{Parametri dell'enunciazione testuale}

Nel caso delle iscrizioni etrusche di dedica l'emittente (con ovvia referenza antroponimica) non coincide necessariamente con il soggetto o l'oggetto sintattici, che nella fattispecie si potrebbero anche definire come il referente "locutivo" (espresso da mi o da mini rispettivamente) ma può apparire con la stessa marca posposizionale $(-s i)$ del ricevente secondo una significativa omologazione pragmalinguistica. Si consideri

TLE 769 (origine incerta, s.d., vaso d'alabastro di genere corinzio) mi licinesi mulu hirsunaiesi

"io da parte di Licine donazione per Hirsunaie"

1 Per il valore di riferimento esoforico $(-n-!)$ dell'isolatissimo turunke di TLE 707 si veda più avanti, nel testo. 
dove si noterà la verbalità non marcata di mulu (non "dono" risultativo, ma "donazione" accadimentale!), l'esitazione "assolutiva" in $-e$ dei due antroponimi, l'assenza in $m i$ referente locutivo e soggetto sintattico di ulteriori indicazioni morfologiche. Diverso è il caso di

TLE 58 (Cere, 600 a.C., su bucchero di forma insolita) mini kaisie $\theta$ annursiannas mulvannice

"Me Kaisie @annursiannas ha dedicato"

dove l'antroponimo corrispondente all'emittente della dedica dell'oggetto svolge la funzione di soggetto sintattico non marcato, l'oggetto parlante è anche oggetto sintattico morfologicamente marcato come pure è marcato il predicato verbale non solo in termini di temporalità (-ce) ma anche in termini che occorre ancora precisare (-anni-), in quanto non riducibili ad una pura e semplice espressione di una funzione denominale (da mulu, v. sopra). ${ }^{2}$

Il ricevente o complemento indiretto, in questo caso con specifica referenza antroponimica, è particolarmente evidente in

TLE 282 (Bomarzo, III sec. a.C., su specchio di bronzo) mi titasi cver menaxe

"Io per Tita (in quanto) dono sono stato fatto"

dove è possibile apprezzare nell'ambito dell'opposizione morfonologica "attivo vs passivo" espressa da $-c / k$ - (in mulvannice del testo precedente) $\mathrm{e}-k h$ - (in menaxe di questo testo) la persistenza dell'esito - $e$ in entrambe le forme verbali che tuttavia hanno soggetti diversi (1' "io" locutivo dell'oggetto parlante e un antroponimo rispettivamente), per cui appare evidente che l'etrusco non marca morfologicamente la persona verbale e che l'esito - e esprime senza distinzione tra antroponimi e verbi quella che abbiamo chiamato condizione o esitazione "assolutiva". Sul contrasto formale tra mi "io" e mini "me", che non è un contrasto morfologico tra nominativo e accusativo, ma un contrasto tra presenza e assenza di enclisi (questa con probabili funzioni di enfasi e probabilmente comparabile con l'infisso -ani- e sue varianti nei verbi), tornerò diffusamente più avanti.

2 Per questa interpretazione, che mi sembra piuttosto riduttiva rispetto a certe evidenze testuali, cfr. Rix 2000, p. 220, ripreso da Facchetti 2008, p. 129. 
L'oggetto dell'azione di offerta, dedica, dono (alpan, cver, fleres, $\vartheta a p / f n a$ e altro ancora) non rientra negli scopi del mio intervento anche perché i riferimenti extralinguistici non sono sempre evidenti o decisivi per le conseguenti scelte lessicali. Allo stesso modo le circostanze accessorie (non predicibili) dovranno essere valutate testo per testo, come nel caso di

TLE 651 (Cortona, III-II sec. a.C., su statua bronzea di uomo nobilissimo detta "l'Arringatore")

auleśi meteliś ve vesial clenśi cen flereś tece sanśl tenine tưineś cisvlicś

"ad Aulo Metello Vesio di Vesio figlio questa statua pose Sanśl Tenine della comunità di (quelli che vengono da) Cisuli (= Chiusi??)"

TLE 652 (Cortona, II sec. a.C., su piccola statua bronzea di fanciullo)

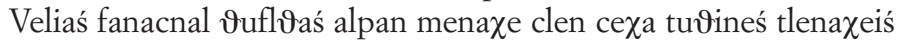

Per Velia Fanacnal Thuflthaś (come) dono sono stato fatto (in quanto immagine di suo) figlio (essendo io/lui?) cekha (sopra?) della comunità di (quelli che vengono da) Tellena"

TLE 363 (Vetulonia, VI sec. a.C. circa, su stele che raffigura un guerriero armato con elmo crestato, scudo rotondo e bipenne)

(a)veleś feluskeś tuśnutn(ie) [...] panalaś mini muluvaneke hirumi(n)a dersnazs

"Ad Aule Feluske. me dedicò Hirumina da Perugia"

In tutti questi testi (e secondo modalità che ho cercato di chiarire altrove), ${ }^{3}$ compare un riferimento morfologico specifico ad un etnico di

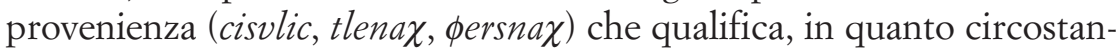
za accessoria non predicibile, l'emittente. In particolare in TLE 651, proprio in quanto iscrizione recenziore (III-II sec.) la forma verbale tece si presenta come nel caso di turuce (v. sopra) senza marcatezza verbale e senza richiamo morfologico alla marcatezza dell'enclisi di $-n$ ravvisabile nel sintagma deittico cen flereśs "questa statua", mentre in TLE 363 (che è di VI sec.!) la sequenza mini muluvaneke conferma la solidarietà morfologica su cui ci siamo concentrati (e di cui si cercherà di fornire un'attendibile spiegazione più avanti).

L'atto di dedica sia esso offerta religiosa o sia altrimenti dono laico

3 Per questa problematica cfr. Silvestri 2005-2006. 
in quanto processualità verbale attiva è espresso da forme come: alice, mulvanice, turuce; in quanto accadimentalità nominale da forme come: aliqu, mulu, turu). Ma le forme qui citate non stanno sullo stesso piano. Infatti aliqu non intrattiene con alice lo stesso rapporto morfologico ravvisabile in turu vs turuce e, a sua volta, muluvanice (a prescindere per ora dalle sue numerose e sconcertanti varianti) introduce, come abbiamo già detto, un terzo tipo di rapporto morfologico con mulu. L'osservazione di Cristofani 1991 "morfologicamente fra aliqu e alice vige la stessa relazione che intercorre fra mulu e mulvanice" (p. 66) è inaccettabile, dal momento che lo stesso Cristofani correttamente riconosce (p. 71) che "esistono in etrusco forme in $-c e$ marcate sia rispetto alle forme in $-u$ sia ad appellativi (cfr. lupu: lupuce, turu: turuce (turce), sval: svalce, zilakb: zilakbce ecc.)" mentre prima (p. 76 dell'edizione del 1973) aveva dichiarato che "il rapporto fra mulu e mulvanice (muluvanice) è un rapporto che va ancora chiarito". D'altra parte lo stesso Cristofani (p. 69), intervenendo sulla coppia verbale muluvene vs muluvenice in due iscrizioni a contenuto identico di VI sec. a.C. (TLE 915: mini muluvene avile acvilnaś; TLE 916: mini muluvenice avi[ile acvil] nas) aveva opportunamente osservato: "Le due iscrizioni comunicano lo stesso contenuto ma non possiamo dire quale tipo di rapporto intercorra tra muluvene e muluvenice". Ad un tentativo di spiegazione in tal senso è dedicato il mio intervento, ma intanto faccio notare che a prescindere da una ipotizzabile base *ale 4 la forma aliqu sembra contenere non solo la stessa esitazione di mulu e di turu ma anche, in termini di marcatezza, una variante (per contiguità con $-u$ ?) del morfema $c / k$ delle forme preteritali (cfr. turuce) per cui la sua esatta traduzione dovrebbe semmai essere "donazione (avvenuta)" e non "donazione (in atto)".

\section{Per un tentativo Di SPIEgazione}

In questa prospettiva passiamo ora in esame le attestazioni di mulu, in quanto nominalità accadimentale, e quelle delle corrispondenti forme "processuali" (cioè le formazioni verbali in $-c e$ )

Attestazioni di mulu

4 Dati reperibili in Pallottino 1954 (= TLE) e in Rix 1991. 
TLE 32 (Monte Soriano, agro falisco, s.d., vaso di argilla rude detto ad "impasto")

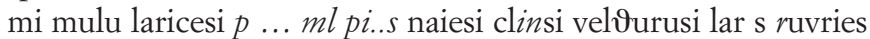

"io donazione a Larice... a Naie figlio a Vel७ur..." (o "da parte di...."?)

TLE 153 (Tarquinii, s.d., askos in bucchero con una figura di gallo)

mi mulu kaviiesi

"io donazione a Kaviie" (o "da parte di Kaviie"?)

TLE 366 (Vetulonia, VII-VI sec. a.C., cyathus in bucchero nel sepolcro "del Duce")

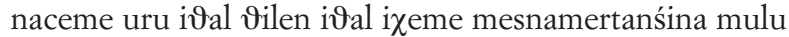

".....................................donazione"

Qui si notino i parallelismi testuali tra naceme e ixeme da una

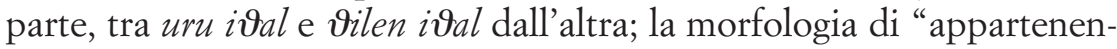

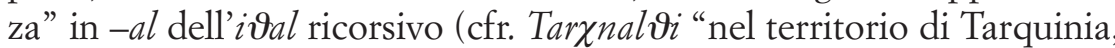
nel Tarquiniese"); la morfologia di "pertinenza" in -na di mesnamertansina.

TLE 759 (origine incerta, s.d., askos in bucchero con una figura di gallo)

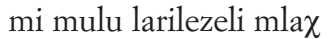

"io donazione per/da parte di/di Larileze buona"

Per la corretta agnizione semantica (basata su un rigorosa evidenza testuale) di mlac si rinvia a Agostiniani 1981, che si è basato sull'equivalenza testuale delle formule DUENOM DUENAS: KA $\mathrm{O} \Sigma$ KA $\Lambda$ Ō: MLAX MLAKAS.

TLE 769 (origine incerta, s.d., vaso d'alabastro di genere corinzio) mi licinesi mulu hirsunaiesi

"io da parte di Licine (sono stato) donato a Hirsunaie"

Per questo testo si rinvia al commento fatto in apertura di discorso a proposito dell'omologia morfopragmatica tra emittente e ricevente.

Attestazioni di muluvanice e varianti

TLE 38 (muleanice): Veio, s.d., oinochoe di bucchero, presenza di mene (ogg.dir.) 
Pallottino si esprime così: "verbi mul... ad integrum restituendi rationem haud certam habeas, cum in titulis Veientanis hoc idem verbum formis admodum variis occurrat"

TLE 506 (mulwen[e]ke): Chiusi, s.d., su tomba, presenza di mi (sogg.), in un contesto dichiarativo di iscrizione "parlante"; poi segue il riferimento al dedicante.

TLE 386 (mulenike): Volterra,s.d., su oggetto imprecisato, presenza di mi (sogg.), come sopra (schema: "io sono di X, Y lo(?) ha dedicato (=riferimento esoforico)

TLE 36 (mulvanice): Veio, s.d., oinochoe in bucchero, presenza di mene (ogg. dir.)

TLE 39 (mulvanice): Veio, s.d., oinochoe in bucchero, presenza di itan ("istud?" ogg.dir.)

TLE 40 (mulvanice): Veio, s.d., oinochoe in bucchero, presenza presumibile di mini (ogg.dir.)

TLE 41 (mulvanice): Veio, s.d., vaso in bucchero, presenza presumibile di mini (ogg.dir.)

TLE 57 (mulvanice): Caere, s.d., anfora in bucchero, presenza di mini (ogg. dir.)

TLE 58 (mulvannice): Caere, s.d., bucchero di forma insolita, presenza di mini (ogg. dir.)

TLE 763 (mulveneke): Or. Inc., s.d., vaso in bucchero, presenza di mini (ogg. dir.)

TLE 725 (mulvenice): Or. Inc., s.d., cippo o lapide, presenza presumibile di mini (ogg.dir.)

TLE 267 (mulvunuke): Volsinii, s.d., oinochoe in bucchero, presenza di mini (ogg.dir.)

TLE 268 (mulvunuke): Volsinii, s.d., oinochoe in bucchero, presenza di mini (ogg.dir.)

TLE 481 (muluevneke): Chiusi, s.d.., vaso in bucchero, presenza di mine (ogg.dir.) in giuntura grafica con viku

TLE 60 (muluemknie): Caere, s.d., askos foggiato geometricamente in forma di anello, presenza di $m i$ (sogg.), in un contesto dichiarativo di iscrizione "parlante"; poi segue il riferimento al dedicante, cfr. TLE 506 e 386

TLE 35 (muluv[an]ece): Veio, s.d., vaso in bucchero, presenza di mini (ogg. dir.)

TLE 363 (mluvaneke): Vetulonia, VII-VI sec. a.C., stele di pietra con immagine di un uomo armato, presenza di mini (ogg. dir.) nella seconda parte del testo, v. sopra

TLE 34 (mulwvanice): Veio, s.d., oinochoe in bucchero, presenza di mini (ogg.dir.)

TLE 40 (muluvanice): Veio, s.d., oinochoe in bucchero, presumibile presenza di mini (ogg.dir.) 
TLE 41 (muluvanice): Veio, s.d., vaso in bucchero, presumibile presenza di mini (ogg.dir.)

TLE 429 (muluvanice): tra Volterra, Chiusi e Arezzo, inizi VI sec. a.C. (?), cyathus in bucchero, presenza di mini (ogg. dir.)

TLE 59 (muluvanike): Caere, s.d., oinochoe in bucchero con figura, presenza di mini (ogg. dir.)

TLE 482 (muluvanike): Chiusi, s.d., poculum in bucchero formato da cinque vasetti, presenza di mini (ogg. dir.)

TLE 407 (muluvanuke): Volterra, s.d., stele con immagine di uomo armato, presenza di $m i$ (sogg.), in un contesto dichiarativo di iscrizione "parlante"; poi segue il riferimento al dedicante, cfr. TLE 60, 386, 506.

La documentazione non è (per ora) esaustiva, ma permette di porre in luce alcune evidenze. In tutti i casi compare la sequenza mini, di cui evidenzio la seconda parte $-n i$, proprio in quanto essa sembra essere morfologicamente ripresa dal morfo in nasale (varianti più ricorsive: -ene-, -eni-, -ani-, -unu-), che compare tra il nucleo designativo di base mulu con marca $-u$ di accadimentalità nominale e la marca di processualità verbale $-c e$. Faccio notare che l'unico caso (TLE 39) di oggetto diretto diverso, consistente in un riferimento deittico esoforico itan, esibisce un'identica condizione morfica. Invece i quattro casi di $m i$ "in distribuzione complementare anche con predicati in $-c e$ " (per usare l'espressione di Cristofani 1991, p.85) si lasciano facilmente inquadrare in una struttura testuale il cui schema è: "io sono di $\mathrm{X}, \mathrm{Y}$ lo(?) ha dedicato". Ma qual è allora la funzione del morfo con nasale (eventualmente intervocalica) che compare in tutti i casi attestati e non ha avuto ancora una spiegazione plausibile? Avanzo l'ipotesi che esso abbia una funzione di riferimento anaforico-deittico all" "io" locutivo ("il qui presente oggetto") e che tale funzione si possa ravvisare, nei termini di un" "enfasi pronominale", 5 anche nel $-n i$ di mini e nel $-n$ di itan e di consimili forme di dimostrativi (cfr. il rapporto ita: itan o itun o ica: ican, rec. $c n$ ) con evidente riferimento esoforico all'oggetto inscritto (la definizione di "accusativo", per altro ristretto ai soli pronomi, è quella canonica, ma è soltanto interpretatio facilior). Se si accede a questa ipotesi, si comprende facilmente l'assenza del morfo nasa-

5 Cfr. Bonfante 2002, p. 83 a proposito di un "emphatic 'definite' accusative" riconoscibile nel sintagma spureni lucairce di TLE 131, che si potrebbe rendere "la città, proprio quella che governò come lucumone”. 
le nelle forme verbali passive in $-\chi e$, dove il riferimento anaforico al topic dell'enunciazione è evidentemente impossibile; e si comprende allo stesso modo il fatto che il morfo in questione non compaia in cop-

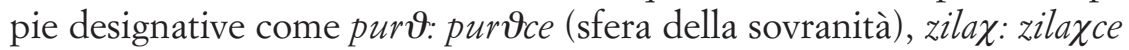
(sfera della magistratura), sval: svalce (sfera della vita), lири: lирисе (sfera della morte) in palese condizione di intransitività. Sull'isolatissimo mi axu muluanix(e) del testo di Caere presente negli Etruskische Texte di Helmut Rix (Cr 3.3) conto di soffermarmi in altra circostanza. Quanto agli aspetti pragmalinguistici di questa specifica documentazione mi limiterò a far notare che queste iscrizioni di dedica sono con prevalenza assoluta su vasellame.

\title{
Attestazioni di turu
}

\author{
TLE 408 (Volterra, s.d., patera in bucchero) \\ menu turu vepet....ś \\ "fatto (e) donato....."
}

La prima parola si presenta con congruenza morfologica rispetto alla seconda e in tal caso potrebbe essere il corrispondente risultativo (accadimentalità nominale, qualcosa come "fattura" e "donazione") dei processuali menece "fece" e menaxe "fu fatto" secondo una modalità testuale del "fare" (che precede fattualmente) e del "donare" (che segue conseguentemente) ben attestata nelle lingue dell'Italia antica (un bell'esempio canonico è nell'iscrizione latinoarcaica della Cista Ficoroni).

TLE 656 (Cortona, ossuario recente) vel pumpu/ś turu a७i/alisa / enicuśi

"da Vel Pumpu donato (lui che è) figlio di Athi a Enicu"

Attestazioni di turce, turke, turuce

(turce)

TLE 149: Tarquinia, s.d., su asta di bronzo, presenza di $c n$

TLE 257: Volsinii, rec., su base, presenza di mi, secondo lo schema testuale della dichiarazione dell'oggetto e della successiva comparsa del dedicante (v. sopra)

TLE 328: Vulci, rec., su candelabro di bronzo, assenza di riferimento all'oggetto

TLE 504: Chiusi, rec., su statua virile, presenza di $\vartheta n$ (cfr. itan?) 
TLE 505: Chiusi, rec., su fallo di bronzo, lacunosa ma con presenza di cver

TLE 557: Chiusi, rec., su piccola statua di bronzo, il dedicante è lautni, un "liberto"

TLE 559: Chiusi, rec., su piccola statua virile in bronzo, presenza di ecn

TLE 610: Perugia, s.d., su herma di pietra, lacunosa, non ulteriormente indagabile

TLE 640: Cortona, IV-III sec. a.C., su piccola statua bronzea di un dio bifronte (dedicatario), presenza di alpan

TLE 641: Cortona, IV-III sec.a C., su piccola statua bronzea di divinità (dedicataria), trovata insieme alla precedente con lo stesso nome di dedicante

TLE 653: Cortona, rec., su piccola statua bronzea di fanciullo, presenza di flereś

TLE 654: Cortona, rec., su candelabro di bronzo, presenza di alpan

TLE 696: Carpegna, rec., su piccola statua virile in bronzo, presenza di $t n$

TLE 726: Or. Inc., ant., su cippo o lapide, presenza di ecn

TLE 736: Or. Inc., rec., su piccola statua bronzea di aruspice, non chiaro riferimento all'oggetto

TLE 739: Or. Inc., rec., su piccola statua bronzea di donna, presenza di flereś

TLE 740: Or. Inc., rec., su piccola statua bronzea con dedica a divinità infernali

TLE 746: Or. Inc., rec., su candelabro di bronzo con immagine della Medusa, presenza di mi, secondo lo schema testuale della dichiarazione dell'oggetto e della successiva comparsa del dedicante (v. sopra)

TLE 752: Or. Inc., rec., su specchio di bronzo con immagini divine, presenza di cver

\section{(turke)}

TLE 622: Perugia, V-IV sec. a.C., sull'ansa di una patera bronzea, nella prima parte del testo presenza di eca

\section{(turuke)}

TLE 156: Tarquinia, kylix attica con figure rosse di stile severo, presenza di itun e dedica ai Dioscuri

TLE 256: Volsinii, ant., su base, assenza di riferimento all'oggetto

TLE 709: Ravenna, VI-IV sec. a.C., su piccola statua bronzea di uomo armato, assenza di riferimento all'oggetto

TLE 743: Or. Inc., su piccola statua di cavaliere in bucchero, assenza di riferimento all'oggetto

TLE 760: Or. Inc., su oinochoe in bucchero, presenza eccezionale di mini

In questo caso il primo fatto di grande portata interpretativa è l'assenza completa sul piano pragmalinguistico del supporto costituito dai 
recipienti e la conseguente assenza sul piano contestuale dello spazio del "privato" (rappresentato da mulvanice) a vantaggio di quello "sacrale e/o pubblico". I recipienti ricompaiono solo con le forme turke (un caso) e turuke (due casi su cinque) in una situazione di possibile sfasamento istituzionale. In tutta la documentazione di turuce ci sono solo due casi di attestazione dell' "io" locutivo, in quella di turuke c'è un solo caso eccezionale di presenza di mini. Questa opzione testuale si risolve in un preciso indice linguistico delle due diverse istanze comunicative (presenza vs assenza della deissi pronominale mi/mini). La situazione sociolinguisticamente elevata è poi confermata nel caso delle attestazioni di turuce dall'assoluta dominanza del supporto bronzeo. Infine nel caso dei testi con turuce va segnalato il carattere decisamente recente della documentazione, mentre i testi con mulvanice sono decisamente più antichi (a questo proposito si deve dare pienamente ragione alle precise puntualizzazioni di Agostiniani 1982, p.200). Sulla base di tutto quanto è stato detto confermo la tesi che il tipo muluvanice (cfr. mulu) è per ragioni morfologiche, testuali e contestuali il più antico e il più autenticamente etrusco, mentre per il tipo turuce (cfr. turu) mi sentirei di sostenere, sempre per ragioni testuali e contestuali, che esso in quanto più recente (e presumibilmente espressione di un acroletto grecizzante) potrebbe trarre origine da gr. $\delta \tilde{\omega} \rho v_{v}$ reinterpretato come risultativo etrusco (turu) e riformulato come processuale etrusco (turuce).

\section{PER UNA POSSIBILE FUNZIONE ANAFORICA DEL MORFO - ANI-/-N- NELLE FORME PERFETTIVE (E TRANSITIVE) DEL TIPO MULUVANICE/TURUNKE}

Come primo indizio a sostegno dell'ipotesi qui proposta ribadisco che il morfo in questione non compare mai nelle forme processuali "intransitive" (tipo lupuce "morì" cfr. lupu "morto") e nelle forme processuali "passive" (tipo menaxe "sono stato fatto" di TLE 282 opposto a menece "fece" di TLE 370, Populonia, s.d., su kylix attica dipinta a figure rosse del genere classico, il cui testo è metru menece con un'assenza doppiamente significativa relativamente all'indicazione dell'oggetto diretto e conseguente non comparsa della qui presunta funzione anaforica di -ani-/-n-). Ulteriori supporti possono in ogni caso venire dall'esame di un'ulteriore documentazione, di cui offro un sintetico assaggio. 
cericun $\chi \mathrm{e} / \operatorname{cercun} \chi \mathrm{e}$

TLE 888 (Cerveteri, IV sec. a.C. circa, iscrizione incisa su pilastro della tomba dei Claudi)

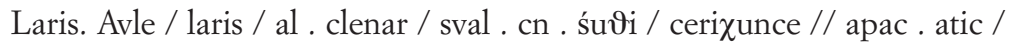
saniśva / ७u / i . cesu // clavtię / urasi

"Laris (e) Avle di Laris figli vivi questa tomba (la?) costruirono. E il padre e la madre morti (?) qui giacciono. Ai Claudii." (tr. di Mauro Cristofani, o.c., p.153)

Si noti che il morfo - ce non registra la concordanza di numero con i due soggetti costituiti dai nomi dei figli. Qui mi limito a far notare la morfologia risultativa di cesu (cfr. turu "donato", mulu "id.", lupu "morto", zilaxnu "(essere stato) magistrato" e, soprattutto, cerixu di TLE 135, dove si parla sicuramente della "costruzione" -ma sarebbe meglio dire "erezione" - di una tomba). Per me cerixu è accadimentalità nominale corrispondente ad un participio passato passivo ed è forma confrontabile con cerine di TLE 315 (eca: śuvic: velus: ezpus / censi: cerine) che a sua volta intrattiene con cerixunce un rapporto paragonabile a quello già riscontrato tra muluvene e muluvenice di TLE 915 e 916 rispettivamente (con l'ovvia avvertenza che il morfo - $\chi$ - di cerizu è espressione allo stesso titolo di temporalità percettiva e di diatesi passiva).

\footnotetext{
TLE 51 (Caere, s.d., su cippo)

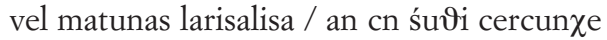

"Vel Matuna di Laris (funzione cataforica di an?) questa tomba (la?) costruì"
}

La forma an è così commentata da Cristofani (o.c., p.87): “an risulta pertanto un elemento che collega la prima alla seconda parte dell'iscrizione, e, nella seconda, sostituisce il soggetto. Troppo poco sappiamo dell'etrusco per poter accostare la funzione di an a quella dei pronomi latini o greci (si è pensato, ad esempio, ai pronomi relativi”. Per la sua probabile funzione anaforica v. dietro.

ur७anike

TLE 764 (origine incerta, s.d., scyphus di bucchero)

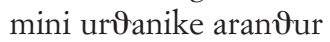

"mi ..... Aran७ur" 
La transitività della forma verbale è assicurata dalla presenza del pronome di prima persona nella forma di "oggetto diretto" (v. sopra); la sua comparabilità morfotattica con muluvanice è tuttavia solo appa-

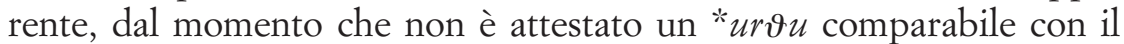
ben documentato mulu e che in ogni caso avrebbe prodotto una plausibile sagoma morfologica * urvuvanike.

\section{turunke}

TLE 707 (Etruria settentrionale, V-IV sec. a.C., su base di pietra) [ar] $\theta$ veiane $\operatorname{sp} u(.$.$) / lariza ma turunke$

"Aronte di Veio (pubblicamente?) ...(lo) ha donato"

In questo caso è anche possibile un riferimento esoforico di $-n$ - al monumento.

\section{CONFRONTI MORFOLOGICI ESTERNI IN AMBITO ANATOLICO ANTICO}

Come secondo indizio a sostegno dell'ipotesi qui proposta segnalo che il morfo in questione ricompare sia in lingue di tradizione non indoeuropea (currito, urarteo) sia in lingue di tradizione indoeuropea (licio, lidio [!]) dell'area anatolica antica, secondo un arco cronologico che copre almeno due millenni, che abbracciano a monte tradizioni grafiche cuneiformi e a valle più e meno recenti repliche alfabetiche. In currito, in particolare, si può registrare la forma enclitica -nna, -n "lui, a lui", che indica sia l'oggetto diretto sia il complemento indiretto e non è separabile -a parer mio- dalla forma tonica anni- "quello" (si noti l'assoluta coincidenza con l'allomorfo -ani- delle forme preteritali etrusche e si ricordi la tipologia agglutinante di questa lingua paracaucasica!). Nell'urarteo, che è forma linguistica recenziore con forti connessioni genealogiche con il currito, è parimenti documentata una forma enclitica $-n i$ con il valore di pronome di terza persona e di oggetto diretto senza distinzione di genere (si noti di nuovo la perfetta coincidenza con l'allomorfo $-n i$ - delle forme preteritali etrusche, che non presentano distinzioni di genere). Il licio, forma recenziore del luvio, ci documenta di nuovo la forma anaforica e enclitica $-n e$ o $-n$ per l'indicazione del pronome accusativo di terza persona singolare e il lidio (quali che siano 
le sue implicazioni protoetrusche) giunge a definitiva conferma con l'enclitico -an, che è forma accusativa di genere comune.

La possibilità che in etrusco sussista una condizione residuale di suffissazione "a catena" in cui, in cui dopo l'indicazione del nucleo designativo verbale compaia prima il riferimento anaforico all'oggetto dell'offerta o della dedica o del dono e poi l'indicazione aspettuale -più che temporale- dell'azione compiuta non è in contrasto con quanto sappiamo circa la sequenzialità morfica dei procedimenti di agglutinazione e aggiunge forse un ulteriore tassello indiziario (in prospettiva paracaucasica) al problema delle origini etrusche e degli antichi viaggi dei Tirreni/Pelasgi (magari con tappa a Lemno) sulle rotte dei metalli verso l'estremo occidente e verso l'emblematica 'I $\tau \alpha \lambda i$ í anch'essa, a suo modo e ab antiquo "terra del fuoco e dei metalli"... "se interpretata val come io dico" (Cfr. Silvestri 2000, 2012). 


\section{BIBLIOGRAFIA}

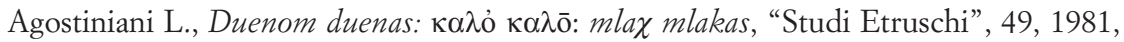
pp.95-111.

Agostiniani L., Le "iscrizioni parlanti" dell'Italia antica, Firenze, Leo S. Olschki, 1982.

Bonfante L., The Etruscan Language, Manchester, Manchester University Press, 2002.

Cristofani M., Introduzione allo studio dell'etrusco, Firenze, Leo S. Olschki, 1991 (nuova edizione interamente aggiornata).

Facchetti G.M., Ancora sull'interpretabilità dell'etrusco: il caso degli specchi, in Mlac mlakas. Per Luciano Agostiniani a cura di Giulio M. Facchetti, Milano, Arcipelago Edizioni, 2008, pp.115-134.

Pallottino M., Testimonia Linguae Etruscae, Firenze, La Nuova Italia, 1954 (ed edizioni successive).

Rix H., Etruskische Texte. Editio Minor, Tübingen, Gunter Narr Verlag, 1991, voll.I-II.

Rix H., La scrittura e la lingua, in Etruschi. Una nuova immagine a cura di Mauro Cristofani, Firenze, Giunti, 2000, pp.199-235.

Silvestri D., Per un'etimologia del nome Italia, “AI $\Omega$ N" 22, 2000, pp. 215-254 (ristampa in "Il mondo enotrio tra VI e V secolo a.C.", Atti dei seminari napoletani (1996-1998) a cura di Maurizio Bugno e Concetta Masseria, Napoli, Loffredo Editore, 2001, pp.207-238 e, con il titolo L'origine greca del nome Italia, in "ITA $\Lambda$ OE $\Lambda \Lambda$ ENIKA Rivista di cultura greco-moderna" 9-10, 2008, pp.185221).

Silvestri D., Etnici di appartenenza ed etnici di provenienza nelle lingue dell'Italia antica, "Studi e Saggi Linguistici" 43-44, 2005-2006 (= Studi in onore di Riccardo Ambrosini), pp.255-269.

Silvestri D., L'Italia prima e oltre Roma. Premesse, storia e destino di un nome in "150 anni. L'identità linguistica italiana”. Atti del XXXVI Convegno della Società Italiana di Glottologia. Testi raccolti a cura di Raffaella Bombi e Vincenzo Orioles. Udine, 27-29 ottobre 2011, Roma, Il Calamo, 2012, pp.29-73. 